Revue internationale P.M.E.

\title{
Partenariats et innovation organisationnelle dans les coopératives agricoles : l'exemple des vins effervescents et des céréales
}

\section{Networking and organisational innovation in agricultural co-ops : cases in sparkling wine and cereals Colaboración e innovación organizacional en las cooperativas agrícolas : el caso de los vinos espumosos e de cereales}

\section{Sophie Reboud, Corinne Tanguy et Michel Martin}

\section{Volume 29, numéro 2, 2016}

URI : https://id.erudit.org/iderudit/1037925ar

DOI : https://doi.org/10.7202/1037925ar

Aller au sommaire du numéro

Éditeur(s)

Editions EMS - In Quarto SARL

ISSN

0776-5436 (imprimé)

1918-9699 (numérique)

Découvrir la revue

Citer cet article

Reboud, S., Tanguy, C. \& Martin, M. (2016). Partenariats et innovation organisationnelle dans les coopératives agricoles : l'exemple des vins effervescents et des céréales. Revue internationale P.M.E., 29(2), 119-144. https://doi.org/10.7202/1037925ar

\section{Résumé de l'article}

Bien que moins étudiée que d'autres formes d'innovation, l'innovation organisationnelle est une composante importante de l'innovation des entreprises, notamment pour celles n'appartenant pas à des secteurs de haute technologie comme c'est le cas des entreprises agricoles et agroalimentaires. Nous nous intéressons plus spécifiquement dans cet article aux coopératives agricoles et à leurs stratégies d'innovation. Trois études de cas nous permettent de montrer comment ces entreprises, plutôt orientées vers des produits bénéficiant de signes de qualité, développent des innovations en s'appuyant sur leur réseau, leurs partenariats, et leurs liens avec leurs adhérents. Cela suppose de leur part des capacités d'absorption et des innovations organisationnelles en complément, voire en substitut, d'innovations technologiques. 


\title{
Partenariats et innovation organisationnelle dans les coopératives agricoles: l'exemple des vins effervescents et des céréales ${ }^{1}$
}

Sophie REBOUD

Sophie Reboud est professeure chercheuse en Stratégie et Management de l'innovation au Groupe ESC Dijon-Bourgogne et chercheuse associée à l'Université d'Australie-Occidentale. Elle s'intéresse depuis plusieurs années aux spécificités des PME et des coopératives de technologie faible à modérée, à leur stratégie et leur innovation et a publié de nombreux articles et ouvrages sur ce thème.

Université Bourgogne Franche-Comté ESC Dijon-CEREN

29, rue Sambin, BP 50608 21006 DIJON CEDEX, France sophie.reboud@escdijon.eu

Corinne TANGUY

Corinne Tanguy est maître de conférences en Économie industrielle à AgroSup Dijon et chercheuse au CESAER, unité mixte de recherche INRA-AgroSup Dijon. Titulaire d'un doctorat en Sciences économiques, elle est spécialiste de l'analyse des réseaux d'innovation en particulier dans les filières agroalimentaires.

Université Bourgogne Franche-Comté UMR1041 CESAER, INRA, AgroSup Dijon 26, bd Docteur Petitjean, BP 87999 21079 DIJON CEDEX, France corinne.tanguy@dijon.inra.fr

Michel MARTIN

Michel Martin, ingénieur INRA, membre du CESAER (Centre d'économie et de sociologie appliquées à l'agriculture et aux espaces ruraux). Ses travaux de recherche sont centrés sur l'analyse des filières agroalimentaires, les coordinations interentreprises et les dynamiques territoriales de l'innovation.

\author{
Université Bourgogne Franche-Comté \\ UMR1041 CESAER, INRA, AgroSup Dijon \\ 26, bd Docteur Petitjean, BP 87999 \\ 21079 DIJON CEDEX, France \\ michel.martin@dijon.inra.fr
}

\footnotetext{
1 Cette recherche a fait l'objet d'une première présentation lors d'un CIFEPME. Nous remercions les évaluateurs du CIFEPME et de la RIPME ayant permis de faire progresser notre réflexion à cette occasion et d'améliorer cet article. Nous restons responsables de toute imperfection qui subsisterait. Cette recherche a été cofinancée par le conseil régional de Bourgogne.
} 


\begin{abstract}
RÉSUMÉ
Bien que moins étudiée que d'autres formes d'innovation, l'innovation organisationnelle est une composante importante de l'innovation des entreprises, notamment pour celles n'appartenant pas à des secteurs de haute technologie comme c'est le cas des entreprises agricoles et agroalimentaires. Nous nous intéressons plus spécifiquement dans cet article aux coopératives agricoles et à leurs stratégies d'innovation. Trois études de cas nous permettent de montrer comment ces entreprises, plutôt orientées vers des produits bénéficiant de signes de qualité, développent des innovations en s'appuyant sur leur réseau, leurs partenariats, et leurs liens avec leurs adhérents. Cela suppose de leur part des capacités d'absorption et des innovations organisationnelles en complément, voire en substitut, d'innovations technologiques.
\end{abstract}

MOTS-CLÉS

Coopérative agricole, Innovation organisationnelle, Coopération, Engagement, Proximité

\title{
Networking and organisational innovation in agricultural co-ops: cases in sparkling wine and cereals
}

\begin{abstract}
Organisational innovations have been less studied than other forms of innovations. They are nonetheless a crucial component of firms' innovations especially for the low-to-mid tech firms, e.g. in agriculture or in the food industry. In this research we focus on co-operatives active in such sectors. We have conducted three exploratory case studies on co-ops developing products with quality labels involving their network, partnerships and the strong link they have built with their members. This suggests that they have developed absorptive capacities and organisational innovations either in complement or as a substitute for technological innovations.
\end{abstract}

KEYWORDS

Agricultural co-op, Organisational innovation, Cooperation, Commitment, Proximity

\section{Colaboración e innovación organizacional en las cooperativas agricolas : el caso de los vinos espumosos e de cereales}

\begin{abstract}
RESUMEN
Aunque menos estudiado que otras formas de innovación, la innovación organizativa es un componente importante de la innovación empresarial, especialmente para aquellos que no desarrollan actividad alta tecnología como es el caso de las empresas agrícolas y agroindustriales. Estamos interesados específicamente en este artículo a las cooperativas agrícolas y sus estrategias de innovación. Tres estudios de caso nos permiten mostrar cómo estas empresas, más orientadas hacia productos que benefician de señales de calidad, desarrollan soluciones innovadoras basadas en redes, asociaciones y relaciones con sus miembros. Esto exige una " capacidad de absorción " y innovaciones organizativas para complementar o incluso sustituir a las innovaciones tecnológicas.
\end{abstract}

Palabras Clave

Cooperativas agricolas, Innovación organizativa, Cooperación, Compromiso, Proximidad 


\section{INTRODUCTION}

Dans une économie dite "de la connaissance », qui met à l'honneur de façon croissante l'innovation, le « modèle particulier $»^{2}$ d'organisation des coopératives pose la question de la capacité de ces entreprises agricoles et agroalimentaires à innover. Les entreprises de ces secteurs innovent plus que celles des autres industries manufacturières (61\% contre $55 \%$ en 2010) (CIS 10). Les innovations organisationnelles, que nous définissons comme «la mise en cuvre d'une nouvelle méthode organisationnelle dans les procédures, l'organisation du lieu de travail ou les relations extérieures de la firme" (OCDE, 2005), sont les plus nombreuses dans ce secteur (Galliano, Garedew et Magrini, 2011). En effet, elles sont déployées par $39 \%$ des entreprises, alors que $31 \%$ des entreprises innovent en matière de produits, et $30 \%$ en termes de procédés ${ }^{3}$.

Notre objectif est donc ici d'appréhender en quoi le fait d'être une coopérative peut influencer positivement le développement d'innovations organisationnelles (Basterretxea et Martínez, 2012), et donc de comprendre comment l'innovation organisationnelle ${ }^{4}$ est mise en ouvre dans les coopératives, en étudiant en particulier les liens de coopération développés par ces entreprises.

Pour cela, nous étudions comment les innovations déployées permettent aux coopératives agricoles d'améliorer leur situation concurrentielle au sein de leur filière. Plus précisément, nous analysons la mise en place d'innovations organisationnelles dans les coopératives se traduisant par la construction et l'évolution de leurs compétences, dans le cadre de la construction de leur avantage concurrentiel (Teece, 1986 ; Barney, 1991). Les innovations de ce type - qui peut prendre la forme d'une nouvelle organisation du travail, de processus de codification et de normalisation qualité, des regroupements entre entreprises (Boehe, 2013) - sont, comme nous l'avons mentionné, nombreuses dans les industries agroalimentaires (IAA), en particulier dans les petites structures, et sont pourtant moins étudiées que les innovations technologiques (Martin et Tanguy, 2011). Pour les coopératives, le choix d'une véritable politique d'innovation s'inscrit dans un contexte d'évolution importante de leurs stratégies face à des enjeux concurrentiels au plan mondial (Levi et Pellegrin-Rescia, 1997 ; Novkovic, 2008 ; Shiraishi, 2009). Ainsi, les stratégies d'alliances et de regroupements au sein de groupes de plus grande taille (Mauget, 2013) constituent pour ces acteurs, comme pour d'autres acteurs de petite taille (Birley, 1985 ; Salavou, Baltas et Lioukas, 2004 ; Street

2 http://www.coopdefrance.coop/fr/15/un-modele-particulier-d-entreprise.

3 Community Innovation Survey (CIS 10, 2008-2010).

4 Selon les enquêtes Community Innovation Survey (CIS), l'innovation organisationnelle se compose de : nouveaux modes de fonctionnement dans l'organisation des procédures (systèmes de gestion de la chaîne d'approvisionnement, reconfiguration des processus, système de gestions des connaissances, production sur commande, systèmes de gestion de la qualité, etc.); nouvelles méthodes d'organisation du travail et de prise de décision (nouvelle répartition des responsabilités, du pouvoir de décision parmi les salariés, travail d'équipe, décentralisation, intégration ou autonomisation de différents services de l'entreprise, systèmes de formation, etc.) ; nouvelles méthodes d'organisation des relations externes avec d'autres entreprises ou organismes (mise en place pour la première fois d'alliances, de partenariats, d'externalisation d'activités ou de sous-traitance). 
et Cameron, 2007), le moyen de construire un nouveau système d'offres grâce à la mise en commun d'actifs spécifiques, le partage d'informations de marché, l'accompagnement de stratégies de diversification ainsi que la maîtrise de l'aval et de la distribution (Chiffoleau et al., 2002 ; D’Angelo, Majocchi, Zucchella et Buck, 2013 ; Esteve-Pérez et Rodríguez, 2013). Ces regroupements permettent en outre, pour les petites coopératives, d'élargir leur gamme de produits, de déployer des efforts marketing et de productivité (Dussuc et Geindre, 2012 ; Fensterseifer et Rastoin, 2013), et de développer des innovations dans de meilleures conditions (Williamson, 1979; Freel, 2003).

Dans une première partie, nous rappelons les spécificités des coopératives agricoles qui facilitent la mise en place d'innovations organisationnelles. En effet, les coopératives ont des liens étroits avec leur territoire d'activité et leurs adhérents, relations qui peuvent se traduire par un engagement important dans la mise en œuvre de changements. Nous montrons comment les coopératives, pour faire face aux nombreuses évolutions auxquelles elles sont confrontées, définissent une stratégie de développement et innovent, en particulier au niveau organisationnel, pour construire des ressources spécifiques. Dans cette perspective, nous nous appuyons sur l'approche développée par les auteurs évolutionnistes (Nelson et Winter, 1982 ; Teece, 1986 ; Dosi, 1988), et par les auteurs de l'approche fondée sur les ressources (Barney et Clark, 2007) qui ont particulièrement analysé le rôle de l'organisation de l'entreprise, ainsi que celui des compétences et des partenariats développés dans une démarche d'innovation. Pour mieux comprendre ces phénomènes, nous mobiliserons aussi la proposition de mise en relation et d'absorption de connaissances externes à l'entreprise (Cohen et Levinthal, 1989, 1990). Nous présentons ensuite dans une seconde partie notre étude empirique basée sur plusieurs études de cas qui témoignent de la façon, dont les innovations organisationnelles permettent d'affronter des situations stratégiques difficiles lors de crises et de déséquilibres soudains. Après une discussion des résultats de ces analyses, nous concluons sur les enseignements que nous pouvons en tirer pour les coopératives, et pour les institutions qui accompagnent leur développement. Ces apports nourrissent également la réflexion en termes académiques sur l'intérêt du modèle coopératif dans un contexte concurrentiel exacerbé et mondialisé comme suggéré par Mangin (2012). 


\section{INNOVATION ORGANISATIONNELLE DANS LES COOPÉRATIVES AGRICOLES ${ }^{5}$ : ASPECTS THEOORIQUES ET CONCEPTUELS}

L’originalité de notre propos est d'examiner comment sont impulsées et développées des innovations de type organisationnel et de chercher à mieux comprendre en quoi le fait d'être une coopérative facilite, ou au contraire rend plus difficile, le fait d'innover. Parmi les changements organisationnels caractéristiques des coopératives, la mise en œuvre des démarches qualité, que ce soit en produit (comme les $\mathrm{AOP}^{6}$ ou l'agriculture biologique) ou en procédé (comme la mise en place d'une norme ISO), très nombreuses dans le secteur agricole et agroalimentaire depuis les années 2000 (Galliano, Garedew et Magrini, 2011), offre un cadre d'analyse très intéressant, car exigeant des changements de pratiques et des innovations de toute nature.

\subsection{Les spécificités des coopératives dans la mise en place des innovations organisationnelles}

Parmi les entreprises dites "de terroir» (Rastoin et Vissac-Charles, 1999 ; Marchesnay, 2001), les coopératives développent de plus en plus une stratégie d'innovation leur permettant de passer d'un statut de " producteur de matières premières » à celui de transformateur allant jusqu'au produit final et intégrant la commercialisation. Pour s’adapter aux évolutions des marchés, les coopératives tendent également à se regrouper en fusionnant ou en constituant une union. Cette politique de regroupement permet de desserrer la contrainte territoriale tout en maintenant un lien fort avec les agriculteurs du territoire et, parfois, facilite le développement à l'international grâce à la mutualisation des ressources (Mauget, 2005). En effet, la nouvelle coopérative (ou l'union) est en mesure de proposer une gamme de produits plus grande aux clients et cela lui permet aussi de se doter d'outils plus performants pour affronter la concurrence sur les marchés national et international (Bélis-Bergouignan, Corade, Couret et Pesme, 2007). Comme nous allons le voir à présent, cette meilleure efficacité concurrentielle permet aux coopératives de mieux valoriser les produits issus des différents terroirs.

5 En France, les 2800 coopératives agroalimentaires de la transformation et du commerce de gros sont articulées autour de trois pôles : le commerce de gros pour un peu moins de la moitié des entreprises, la vinification pour un tiers et la fabrication de fromages pour $10 \%$ (Ambiaud, 2009). Par ailleurs, le poids économique des coopératives est très faible par rapport à l'ensemble des entreprises du secteur agroalimentaire, car un grand nombre de ces entreprises sont des structures de petite taille. En 2012, près des deux tiers comptent moins de dix salariés et près de $80 \%$ moins de vingt, chiffres qui n'évoluent que faiblement depuis dix ans. Source : INSEE LIFIUS, issu de l'enquête des liaisons financières (LIFI) et du fichier approché des résultats Esane (élaboration des statistiques annuelles d'entreprise), enquête sur les liaisons financières entre sociétés (LIFI, 2012).

6 AOC/AOP : l'appellation d'origine contrôlée (AOC) désigne un produit dont toutes les étapes de fabrication sont réalisées selon un savoir-faire reconnu dans une même zone géographique, qui donne ses caractéristiques au produit. L'appellation d'origine protégée (AOP) est l'équivalent européen de l'AOC. Elle protège le nom d'un produit dans tous les pays de l'Union européenne. 


\subsubsection{QUEL CONTEXTE POUR L'INNOVATION DANS LES COOPÉRATIVES?}

Contrairement aux entreprises de droit commercial, les coopératives ont comme spécificité de devoir gérer les contraintes liées à un périmètre d'action imposé et à leur inscription territoriale, tout en valorisant la production des adhérents (Filippi, 2004 ; Filippi et Triboulet, 2006). Si la mutualisation de l'offre en amont des filières peut se révéler un avantage certain, le fonctionnement des coopératives et leurs liens privilégiés avec leurs adhérents leur donnent également un atout quant à la mise en place de nouveaux projets comme les démarches qualité. D’après la littérature (Filippi, 2004), les coopératives agricoles de production présentent un certain nombre de caractéristiques, les amenant ces dernières années à d'importantes évolutions organisationnelles :

- Une offre contrainte par la production fournie par les producteurs adhérents, avec une forte orientation «offre » et une faible orientation « marché ». Cependant, si à l'origine la coopérative multimétiers (lait, viande, légumes, cidre, jardineries...) vendait des engrais et des machines agricoles, la moitié de son chiffre d'affaires provient aujourd'hui de la transformation de ses matières premières. De nombreuses coopératives choisissent en effet d'intégrer dans leurs activités, via des filiales, la transformation de productions agricoles, et de développer des marques pour résister à la grande distribution.

- Un encastrement territorial et culturel : tout d'abord, les produits vendus par la coopérative constituent fréquemment une partie de la culture gastronomique locale, souvent « institutionnalisée » dans des signes de qualité comme les AOC/AOP (appellation d'origine contrôlée ou protégée) qui imposent un cahier des charges et des conditions de production très précises ${ }^{7}$; la contrepartie de cet engagement fort dans des signes de qualité serait un attachement des producteurs membres de la coopérative à leurs savoirs et savoir-faire traditionnels et ils pourraient à ce titre être réticents à les modifier (Gade, 2004).

- Une stratégie qui s'inscrit dans le long terme : une autre spécificité des coopératives est qu'elles semblent, par certains côtés, mieux armées pour résister à la crise économique actuelle $^{8}$, entre autres parce que les excédents dégagés sont essentiellement utilisés pour investir afin d'améliorer l'outil de production et/ou de commercialisation.

Les coopératives agricoles, du fait de leur statut, ont un lien fort avec leurs adhérents sur leur territoire. Ce lien est encore renforcé pour les coopératives qui sont situées dans une zone AOC/AOP (Filippi et Triboulet, 2006). Tout l'enjeu des entreprises coopératives est d'être ainsi à la fois capables de maintenir, voire de renforcer, des liens étroits avec leur territoire et leurs producteurs, mais aussi de s'affranchir de ce territoire pour aller chercher si nécessaire des compétences pour innover qu'elles ne maîtrisent pas en interne, auprès de partenaires localisés hors de cette zone.

7 Ainsi, d'après le site Internet de Coop de France : les entreprises coopératives agricoles jouent depuis longtemps un rôle moteur dans $30 \%$ des Labels rouges, $45 \%$ des CCP (certificats de conformité produits), et dans les AOC qui concernent, par exemple, $80 \%$ des caves coopératives viticoles (Source : http://www.coopdefrance.coop/fr/index.html).

8 Voir à ce sujet la tribune de P. Mangin, président de l'alliance des coopératives agricoles françaises, dans Le Monde du 5 mars 2012 : les coopératives agricoles sont "indélocalisables ", elles sont les champions du savoir-faire français qui peut s'exporter, elles inscrivent leur gestion dans le long terme et sont, par leur origine même, conçues pour résister aux crises. 


\subsubsection{LE RÔLE DES CONNAISSANCES EXTERNES ET DE L'INSERTION DANS DES RÉSEAUX DE COOPÉRATION}

Selon les auteurs évolutionnistes (Nelson et Winter, 1982 ; Dosi, Teece et Winter, 1990), l'innovation est indissociable d'une modification des compétences organisationnelles existantes, modification qui peut s'avérer difficile à mettre en œuvre. David Teece identifie la compétence foncière d'une firme comme la base de ses capacités concurrentielles dans une activité particulière (Teece, 1988) : "Ces gens sont bons en matière de... résume les perceptions extérieures quant à la nature de ces compétences " (Dosi, Teece et Winter, 1990). La compétence foncière est identifiée aux compétences technologiques d'une firme, aux actifs complémentaires et aux routines, le concept de « routine » faisant ici référence à l'analyse de Richard Nelson et de Sydney Winter exposée dans leur ouvrage de 1982. Dans cette optique, la dynamique innovante d'une entreprise n'est pas uniquement déterminée par le montant des investissements matériels et immatériels qu'elle consent, mais elle dépend également de ses compétences et de leurs évolutions, notamment organisationnelles. La compétence est ainsi non seulement considérée comme un acquis, mais également comme une capacité de renouvellement et de changement (Divry, Dubuisson et Torre, 1999). L'élaboration de nouvelles pratiques et le développement de nouveaux produits peuvent rencontrer des obstacles dans la mesure où les savoirs, qui sont à la base de la conception des produits et de la gestion des procédés, sont encastrés dans les compétences existantes ou les routines de la firme. Pour autant, la construction dans le long terme d'un avantage spécifique repose sur l'accumulation de connaissances fondant la différenciation et pouvant servir de levier à une innovation (Teece, 1998).

Dans cette optique, un aspect primordial du processus d'innovation est la capacité d'une firme à exploiter les connaissances externes, autrement dit sa capacité d'absorption (Cohen et Levinthal, 1989, 1990). En fonction de cette capacité, une entreprise sera plus ou moins en mesure d'exploiter les opportunités technologiques de son environnement (Martin et Boschma, 2010). La capacité à « absorber » les technologies et connaissances externes (à mobiliser les ressources externes, existantes) dépend alors de la manière, dont elle est organisée en interne (circulation de l'information, dispositifs de veille technologique, existence de service R\&D et/ou qualité) (Ter Wal et Boschma, 2011). Elle dépend aussi de la manière, dont elle est organisée vis-à-vis de son environnement extérieur, c'est-à-dire, dont elle est insérée dans des réseaux d'innovation. Dans cette perspective, et comme l'ont montré Giuliani et Bell (2005) dans leur analyse du cluster vitivinicole dans une région du Chili, le degré d'ouverture des entreprises aux connaissances et appuis extérieurs apparaît fondamental. Les petites structures peuvent dans certains cas jouer un rôle clé de facilitateur d'innovation, assumant, à la place des grandes entreprises, un risque qui n'est pas sans danger pour elles (Puthod et Thévenard-Puthod, 2006). Les coopératives doivent ainsi être capables d'intégrer des connaissances externes et de s'insérer, y compris à l'extérieur de leur territoire coopératif, dans des réseaux de relations pour innover. Or, la familiarité avec des habitudes de coopération, comme c'est le cas dans les coopératives, peut réduire ce danger et se révéler un facilitateur d'innovation (Cohen et Levinthal, 1990). À cet égard, la proximité géographique ne semble pas être le seul facteur favorisant l'innovation. En effet, la coordination entre acteurs repose ainsi sur d'autres facteurs que la seule colocalisation et proximité géographique 
(Ben Letaifa et Rabeau, 2013), tels qu’une proximité organisée ${ }^{9}$ fondée sur des normes collectives et des codes communs de communication qui stabilisent le système de relations et rendent la collaboration possible (Torre et Rallet, 2005 ; Zimmermann, 2008 ; Torre, 2010). L'existence d'un langage et de normes communes constitue ainsi un élément moteur dans les démarches d'innovation et l'engagement des coopérateurs.

\subsubsection{MOBILISATION DES ADHÉRENTS ET DÉMARCHES D'INNOVATION}

Que ce soit pour intégrer la commercialisation des produits, ou pour en certifier l'origine ou les conditions de production, les innovations organisationnelles développées par les coopératives s'appuient sur les relations privilégiées avec leurs adhérents. En effet, les certifications basées sur une meilleure valorisation des liens au territoire, à travers le développement de signes de qualité et d'origine, sont difficiles à mettre en place et exigent la création de relations partenariales entre l'entreprise et ses adhérents (Stervinou et Lê, 2006). Or, le partage d'informations et la communication interne au sein des coopératives se font plus facilement que dans des entreprises classiques (Arcas-Lario et Hernandez-Espallardo, 2003 ; Birchall, 2011). La prise de décision collective, le partage d'une culture commune et le développement de routine de collaboration favorisent donc l'innovation (Bourgeois et Brodwin, 1984; Chatenier, Verstegen, Biemans, Mulder et Omta, 2010) montrant ainsi l'importance, dans le processus collectif de création de nouveaux savoirs et de nouveaux savoir-faire, de la création de confiance, d'engagement et d'une culture partagée.

Facteur primordial d'échanges d'informations et de connaissances en interne et en externe, les changements de pratiques et l'innovation organisationnelle jouent ainsi un rôle central dans la performance des coopératives, mais ces phénomènes restent relativement peu étudiés, l'essentiel des travaux sur l'innovation des entreprises en général, et des PME en particulier, s'intéressant traditionnellement plutôt à l'innovation technologique. Or, les interrelations entre les innovations technologiques et organisationnelles sont nombreuses (Lam, 2004 ; Ayerbe-Machat, 2006 ; Fonrouge, 2008) et leur prise en compte est essentielle à la réussite des projets d'innovation. En effet, très souvent, une innovation va entraîner des modifications d'ordre technologique, mais aussi des changements dans l'organisation, et nécessiter par conséquent une évolution des compétences. Ces innovations nécessitent de nouvelles coordinations (Allaire et Sylvander, 1997 ; Filippi et Triboulet, 2006) et les liens privilégiés des coopérateurs entre eux, et avec leur coopérative, favorisent une mobilisation des adhérents dans les actions collectives.

9 Selon les tenants de l'école de la proximité, la proximité organisée rend compte du fait que les partenaires de la coopération vont partager des règles communes en termes d'organisation, de représentations et de règles d'action qui leur permettent de se coordonner. Le fait d'être localisés à proximité ne suffit pas pour engendrer une action coordonnée des acteurs avec leurs voisins proches (Zimmermann, 2008). 


\subsection{Le défi coopératif actuel : coopérer et innover tout en maintenant des liens étroits avec leurs adhérents}

Pour faire face aux nombreuses évolutions auxquelles elles sont confrontées et définir une stratégie de développement, les coopératives doivent ainsi être capables d'innover, en particulier au niveau organisationnel, pour construire avec leurs adhérents des ressources spécifiques. Cependant, plusieurs auteurs (Fulton, 1999 ; Barraud-Didier et Henninger, 2009 ; Barraud-Didier, Henninger et Anzalone, 2013) ont montré que l'engagement des coopérateurs tend à diminuer avec l'accroissement de la taille de la coopérative. Une étude récente menée sur le processus de participation des adhérents de coopératives en Midi-Pyrénées montre en effet que, plus la coopérative est grande et complexe, moins les adhérents se sentent « engagés » dans la coopérative et s'impliquent dans sa gouvernance (Barraud-Didier, Henninger et Triboulet, 2014). Les restructurations et le développement de stratégies commerciales plus offensives provoquent également un éloignement des adhérents de la coopérative qui peuvent se trouver de ce fait moins « engagés » vis-à-vis de leur coopérative (Fulton, 1999 ; Barraud-Didier et Henninger, 2009 ; Barraud-Didier, Henninger et Anzalone, 2013). Cela ne signifie pas cependant qu'ils remettent en question leur participation économique. En revanche, ils sont plus susceptibles d'adopter des comportements opportunistes d'adhésion/non-adhésion en fonction des variations de conditions de marché et de contexte. Les coopératives semblent conscientes de cette volatilité de l'attachement de leurs membres et cherchent à maintenir les conditions d'un attachement durable.

La question que nous nous posons est celle de l'influence de la spécificité des entreprises coopératives sur le développement d'innovations organisationnelles, en particulier à travers la mise en œuvre de coopérations. L'analyse des stratégies de partenariat des coopératives spécialisées dans l'élaboration de Crémant de Bourgogne, de Champagne et de céréales biologiques va à présent nous permettre de préciser les innovations organisationnelles qui ont été développées dans les coopératives, à l'occasion de la mise en place de démarches de régulation de la production et d'amélioration de la qualité des produits.

\section{MÉTHODOLOGIE}

\subsection{Choix des cas étudiés et collecte des données}

Notre objectif est d'explorer les spécificités de la mise en œuvre des innovations organisationnelles menées par les coopératives en réalisant des études de cas de type instrumental dans deux secteurs de l'agroalimentaire (vins effervescents et céréales). D’après Chetty (1996), la méthodologie de recherche par étude de cas est une forme de recherche essentielle en sciences sociales et en management, aussi bien pour tester des hypothèses (Yin, 1989) que pour explorer et développer de nouvelles théories (Eisenhardt, 1989). Un des avantages reconnus à cette méthodologie est qu'elle permet de mesurer et repérer des comportements, au contraire de méthodes par entretiens qui ne capturent que des déclarations verbales (Yin, 1989). Elle autorise aussi le recueil d'informations de sources multiples, qualitatives comme quantitatives (Chetty, 1996). Ainsi, ce type de méthode permet grâce à l'utilisation de mul- 
tiples sources d'informations de fournir une image globale beaucoup plus complexe et riche que d'autres méthodes (Sohal, Simon et Lu, 1996). Parmi les critiques qui lui sont faites, la faible possibilité de généralisation est réfutée par Yin (1989) qui compare les études de cas à des expériences, qui présenteraient un potentiel de généralisation à des propositions théoriques et non à des populations ou à des univers (Chetty, 1996).

Nous avons privilégié cette méthodologie dans le cadre d'une démarche exploratoire qui ne cherchait pas à valider une proposition théorique, mais plutôt à mieux comprendre les dimensions de la construction de compétences pour innover au niveau organisationnel par les coopératives. Ces études de cas sont de nature descriptive et nous cherchons à améliorer la compréhension du phénomène que nous étudions (Charreire et Durieux, 1999).

Les cas étudiés ont été choisis dans deux secteurs dans lesquels les coopératives sont particulièrement représentées : la vinification, et plus particulièrement la fabrication de vins effervescents, et le commerce de gros de céréales (Ambiaud, 2009).

La collecte de données a été permise par l'activité pédagogique de deux des auteurs, les amenant à entretenir une relation fréquente avec les coopératives étudiées. Dans le cadre de la supervision d'étudiants, ils ont pu directement collecter des données. Ces données primaires ont été complétées par l'analyse de données secondaires issues de la presse (Tableau 1).

Tableau 1. Synthèse des différentes Collectes de donnéES

\begin{tabular}{|c|c|c|c|}
\hline \multirow[t]{2}{*}{ Cas } & \multirow[t]{2}{*}{ Données secondaires } & \multicolumn{2}{|l|}{ Données primaires } \\
\hline & & $\begin{array}{l}\text { Collecte par étude de cas } \\
\text { groupe d'étudiants }\end{array}$ & Autres modes de collecte \\
\hline \multirow[t]{2}{*}{ Crémant } & \multirow[t]{2}{*}{$\begin{array}{l}\text { Fournies par l'Union } \\
\text { des producteurs } \\
\text { et élaborateurs de } \\
\text { Crémant de Bourgogne, } \\
\text { complétées par des } \\
\text { rapports, études, articles } \\
\text { scientifiques et données } \\
\text { provenant des organismes } \\
\text { statistiques publics. }\end{array}$} & $\begin{array}{l}\text { Six semaines, } \\
23 \text { étudiants (trois } \\
\text { groupes d'étudiants : } \\
\text { l'un enquêtant sur les } \\
\text { viticulteurs, le deuxième } \\
\text { les élaborateurs de } \\
\text { Crémant et le troisième: } \\
\text { les distributeurs et } \\
\text { consommateurs). }\end{array}$ & $\begin{array}{l}\text { Mémoire de fin } \\
\text { d'études d'ingénieur } \\
\text { de six mois sur la } \\
\text { question de l'innovation } \\
\text { (technologique et } \\
\text { organisationnelle) dans } \\
\text { la filière Crémant de } \\
\text { Bourgogne. }\end{array}$ \\
\hline & & $\begin{array}{l}\text { Enquêtes directes (onze } \\
\text { élaborateurs privés, onze } \\
\text { coopératives), entretiens } \\
1 \text { h } 30 \text {, questions } \\
\text { ouvertes, traitement } \\
\text { qualitatif. }\end{array}$ & $\begin{array}{l}\text { Enquêtes directes (onze } \\
\text { experts, } 19 \text { élaborateurs } \\
\text { (dix coopératives, neuf } \\
\text { privés), entretiens } 1 \text { h } 30 \\
\text { à } 2 \mathrm{~h} \text {, semi-directifs, } \\
\text { qualitatifs. }\end{array}$ \\
\hline
\end{tabular}




\begin{tabular}{|c|c|c|c|}
\hline Cas & Données secondaires & Données primaires & \\
\hline \multirow[t]{2}{*}{ Champagne } & \multirow{2}{*}{$\begin{array}{l}\text { Informations et données } \\
\text { disponibles dans une } \\
\text { thèse, et des articles de la } \\
\text { presse professionnelle. }\end{array}$} & & $\begin{array}{l}\text { Mémoire de fin d'étude } \\
\text { d'une étudiante en } \\
\text { formation d'ingénieur. }\end{array}$ \\
\hline & & & $\begin{array}{l}\text { Enquêtes directes (treize } \\
\text { viticulteurs + entretiens } \\
\text { avec l'Union Auboise et } \\
\text { coopératives de l'Union). }\end{array}$ \\
\hline \multirow[t]{2}{*}{ Céréales } & \multirow{2}{*}{$\begin{array}{l}\text { Synthèse d'articles de } \\
\text { recherche, d'articles de } \\
\text { presse professionnelle } \\
\text { en veillant à croiser les } \\
\text { sources d'informations } \\
\text { pour vérifier les } \\
\text { informations ou } \\
\text { données que nous avons } \\
\text { mobilisées. }\end{array}$} & $\begin{array}{l}\text { Étude de cas groupe } \\
\text { d'étudiants ( } 21 \text { étudiants, } \\
\text { six semaines, trois } \\
\text { groupes : un enquêtant } \\
\text { les stockeurs, un les } \\
\text { agriculteurs et un les } \\
\text { distributeurs). }\end{array}$ & $\begin{array}{l}\text { Nombreuses discussions } \\
\text { avec une coopérative, } \\
\text { acteur central de la filière } \\
\text { céréales, en vue d'un } \\
\text { dépôt d'un projet financé } \\
\text { par le Fonds unique } \\
\text { interministériel (FUI). }\end{array}$ \\
\hline & & $\begin{array}{l}\text { Enquêtes directes (sept } \\
\text { organismes stockeurs, } \\
\text { quatre stockeurs/ } \\
\text { meuniers, } 1 \text { h } 30 \text {, } \\
\text { + agriculteurs et } \\
\text { distributeurs). }\end{array}$ & $\begin{array}{l}\text { Entretiens avec dirigeants } \\
\text { de coopératives venant } \\
\text { compléter les autres } \\
\text { sources. }\end{array}$ \\
\hline
\end{tabular}

\subsection{Adhésion des producteurs, compétences et innovation : trois études de cas}

Les coopératives, dans le secteur des vins effervescents, développent une stratégie de sécurisation des approvisionnements ${ }^{10}$ et d'amélioration de la valorisation des raisins de leurs adhérents. Traditionnellement, les coopératives sont les fournisseurs de raisin ou de vin de base aux élaborateurs, mais depuis quelques années, elles intègrent de plus en plus l'étape d'élaboration et développent leurs propres marques. Dans la filière céréales, la Cocebi a été pionnière dans la constitution d'une filière « agriculture biologique » en structurant une nouvelle organisation de la production et de la distribution. Aujourd'hui, en s'associant avec d'autres acteurs (Tableau 2), elle cherche à développer de nouveaux marchés.

Pour mettre en place ces stratégies, les coopératives innovent aux plans technologique et organisationnel. Dans la mise en œuvre de ces démarches d'innovation, nous analysons le rôle des modifications de procédures et des coopérations externes, ainsi que celui que jouent les relations privilégiées que les coopératives entretiennent avec leurs adhérents.

10 Pierre du Couédic, délégué général de l'UPECB, l'Union des producteurs et élaborateurs de Crémant de Bourgogne déclare en 2014 : « Le Crémant de Bourgogne est dans une situation tendue : les récoltes ne suffisent pas à répondre à la demande. Nous risquons de perdre des marchés, par manque de produit alors que le Crémant de Bourgogne est en pleine croissance ». Source : http://www. viteff-blog.com/un-contingent-de-plantation-pour-le-cremant-de-bourgogne. 


\subsubsection{LES COOPÉRATIVES PRODUCTRICES DE VIN EFFERVESCENT ${ }^{11}$ : Champagne et Crémant de Bourgogne}

Situées dans des régions voisines et mettant en œuvre les mêmes procédés de fabrication, les coopératives productrices de vin effervescent Champagne et Crémant poursuivent un même objectif de fidélisation des adhérents et d'innovations organisationnelles destinées à résoudre la question cruciale de l'approvisionnement, en qualité, régularité et quantité, de la matière première employée : le raisin.

\section{L'Union Auboise : l'innovation organisationnelle au service de sa stratégie de différenciation}

Même le Champagne, considéré comme une des appellations les plus prestigieuses du monde, peut connaître de façon cyclique, une crise qui entraîne une diminution des ventes, notamment à l'export, ou une diminution des prix sur le marché national et à l'export. En 2008, cette baisse, particulièrement notable pour les maisons de négoce les plus exportatrices, a eu une conséquence indirecte, celle de remettre en question l'équilibre organisationnel instauré depuis des décennies entre les vignerons, producteurs de raisin et de matières premières, qui, pour $70 \%$ d'entre eux, sont adhérents de coopératives, et les maisons de négoce qui élaborent le Champagne et en assurent la distribution ( $70 \%$ de la commercialisation des bouteilles finies et $90 \%$ sur les marchés extérieurs hors UE) et qui font la notoriété du Champagne.

Le lien entre les vignerons et les négociants se fait par l'intermédiaire de leurs organismes professionnels respectifs qui siègent ensemble dans un organisme interprofessionnel, qu'ils codirigent : le Comité interprofessionnel des vins de Champagne (CIVC). Celui-ci, depuis sa création en 1941, est responsable de la mise en œuvre des contrats qui, même s'ils ont évolué au cours du temps, ont toujours pour fonction de réguler le marché et l'équilibre entre les différents acteurs champenois. Ainsi, l'interdépendance des deux familles, les vignerons et les négociants, est à l'origine de "l'équilibre champenois » qui a assuré jusqu'à présent le partage de la valeur ajoutée de manière équitable (CIVC, 2010).

En 2008-2009, certains marchés à l'export connaissent une baisse pouvant aller jusqu'à $35 \%$ des volumes, ce qui engendre une diminution des prix en France comme à l'étranger. Afin de limiter les impacts de la crise, certaines maisons de Champagne annoncent qu'elles ne peuvent pas s'engager comme à l'accoutumée sur des contrats de quatre ans et qu'un accord ne sera trouvé que dans le cadre d'une diminution des rendements afin de réduire les stocks et le prix du raisin. Cette pression est alors subie par les vignerons comme un moyen pour les maisons de Champagne de se protéger de la crise au détriment des viticulteurs, remettant en cause l'équilibre partenarial établi.

L’Union Auboise (UA) est une union de coopératives créée en 1967 par les onze coopératives viticoles de l'Aube. L'idée, à l'époque, était de construire une organisation constituant un outil d'élaboration de Champagne et de commercialisation commun, et de conserver une part plus importante de la valeur ajoutée. Aujourd'hui, l'Union Auboise regroupe douze coopératives, indépendantes et menant des activités complémentaires, et 900 adhérents. Il s'agit de l'un des

11 Le vin effervescent le plus connu est le Champagne, qui a donné son nom à la méthode de production dite «méthode champenoise». Lorsque le vin n'est pas effervescent, il est dit « tranquille». Ce dernier est le plus répandu. 
opérateurs majeurs de la région. Outre une production à destination des grandes maisons de négoce, l'Union Auboise commercialise du Champagne sous sa propre marque (marque Devaux, sa marque emblématique) et sous des marques (Jacquart, Montaudon) détenues par Alliance Champagne, une union constituée avec deux autres unions de coopératives : la Cogevi et la Covama, localisées respectivement dans les départements de la Marne et de l'Aisne.

La course à l'approvisionnement étant très concurrentielle en Champagne, les adhérents des coopératives sont très convoités. L’Union Auboise a donc mis en place différentes stratégies de fidélisation auprès des coopérateurs. Le service développement de l'Union Auboise est responsable du maintien et de l'augmentation du sociétariat au sein des différentes coopératives. Le service technique offre un accompagnement aux adhérents dans les pratiques réglementaires, environnementales et qualitatives sur les exploitations dans le cadre d'une démarche volontaire proposée aux adhérents, la Démarche Qualité Vignoble (DQV). Une telle démarche, analysée au niveau de l'UA, a constitué une innovation organisationnelle lors de sa mise en place.

Suite à la crise de 2008 et au conflit qui a opposé les familles de viticulteurs et de négociants au sein de l'interprofession (CIVC), une des stratégies de l'Union Auboise a ainsi été de trouver un levier de différenciation pour accentuer sa politique de marque. L’objectif était de devenir un partenaire incontournable et de ne pas dépendre trop fortement des ventes auprès des maisons de Champagne qui absorbent la moitié de la production de l'union de coopératives. La Démarche Qualité Vignoble, destinée à améliorer les pratiques qualitatives et respectueuses de l'environnement, et mise en œuvre dans les exploitations volontaires depuis les années 2000 (184 sur les 900 adhérents pour une surface de 1000 ha en 2010), est apparue ainsi comme une démarche à même de procurer un avantage concurrentiel à l'Union Auboise, à condition que cette qualité soit perçue et valorisée auprès des consommateurs. En effet, les exigences des consommateurs en termes de qualité de produits, de qualité sanitaire et de respect de l'environnement ainsi que l'évolution conjointe de la réglementation (Plan Ecophyto 2018, directives Nitrates, etc.) obligent les acteurs de la filière champenoise à s'adapter et à développer de nouvelles pratiques. Les études montrent cependant que cet effort d'adaptation et de mise en place des nouvelles modalités organisationnelles que recouvre cette " Démarche Qualité Vignoble " est encore en voie de réalisation et qu'il aura nécessité un apprentissage de plusieurs années. Comme dans d'autres démarches d'innovation, la diffusion de la DQV s'est faite progressivement, en s'appuyant sur les acteurs les moins réticents (Akrich, Callon et Latour, 1988 ; Rogers, 1995). Le développement de ces nouveaux itinéraires techniques de conduite de la vigne et de nouvelles procédures d'organisation a d'abord été proposé aux quelques volontaires, reconnus comme des «pionniers » dans la coopérative, avant d'être progressivement étendu aux autres coopérateurs.

Cette démarche de production durable permet à la filiale de la coopérative, l'entreprise Devaux $^{12}$, de s'approvisionner en raisins de qualité et permet ainsi d'obtenir un avantage concurrentiel vis-à-vis des autres maisons champenoises. Ainsi, ses ventes croissent en 2013 de $15 \%$ en volume. L'exportation a représenté $50 \%$ de ses expéditions.

12 Le président de la coopérative déclare : "Nous sommes dans une logique de marque "boutique" basée sur la valeur plus que sur le volume » : http://www.lopinion.fr/28-novembre-2014/l-unionauboise-monte-en-gamme-devaux-18872. 


\section{Le rôle moteur des coopératives dans le développement de la filière Crémant de Bourgogne}

L'AOC Crémant de Bourgogne, vin effervescent, a été reconnue officiellement en 1975. Elle est gérée par l'Union des producteurs et élaborateurs de Crémant de Bourgogne (UPECB), qui regroupe environ 240 viticulteurs, caves coopératives et élaborateurs, des départements de la Côte-d'Or, du Rhône, de la Saône et Loire et de l'Yonne. La commercialisation de Crémant de Bourgogne est en croissance continue depuis sa création. Au sein de la filière AOC Crémant de Bourgogne, les coopératives jouent un rôle stratégique en tant que fournisseur de vin de base qui va ensuite être transformé en crémant.

Les entreprises privées réalisent l'essentiel de leur chiffre d'affaires dans l'élaboration de Crémant de Bourgogne en achetant des raisins, des moûts ${ }^{13}$ ou du vin de base auprès des viticulteurs ou des coopératives. Les coopératives sont, quant à elles, plutôt orientées vers la production de vins tranquilles ${ }^{14}$ et se diversifient en produisant des vins de base. Les coopératives présentes dans la filière crémant sont des structures de petite taille, avec en moyenne seize salariés (quatre pour la plus petite et 50 pour la plus grande). Seule la plus grande est spécialisée dans l'élaboration de crémant, elle y réalise $80 \%$ de son chiffre d'affaires et maîtrise l'ensemble du processus de production (Figure 1). Les autres coopératives privilégient la production de vins tranquilles et la production de vin de base avec une tendance à développer de plus en plus une production en propre de crémant. Pour ces coopératives, la production de crémant représente en moyenne 11 à $12 \%$ de leur chiffre d'affaires. En règle générale, elles délèguent les phases d'élaboration du crémant à d'autres entreprises du fait des équipements et des savoir-faire nécessaires, et seules deux coopératives ont choisi d'internaliser l'ensemble des phases de processus de production du crémant.

Figure 1. Division fonctionnelle du travail aU SEIN DE LA Filière Crémant DE BOURGOGNE

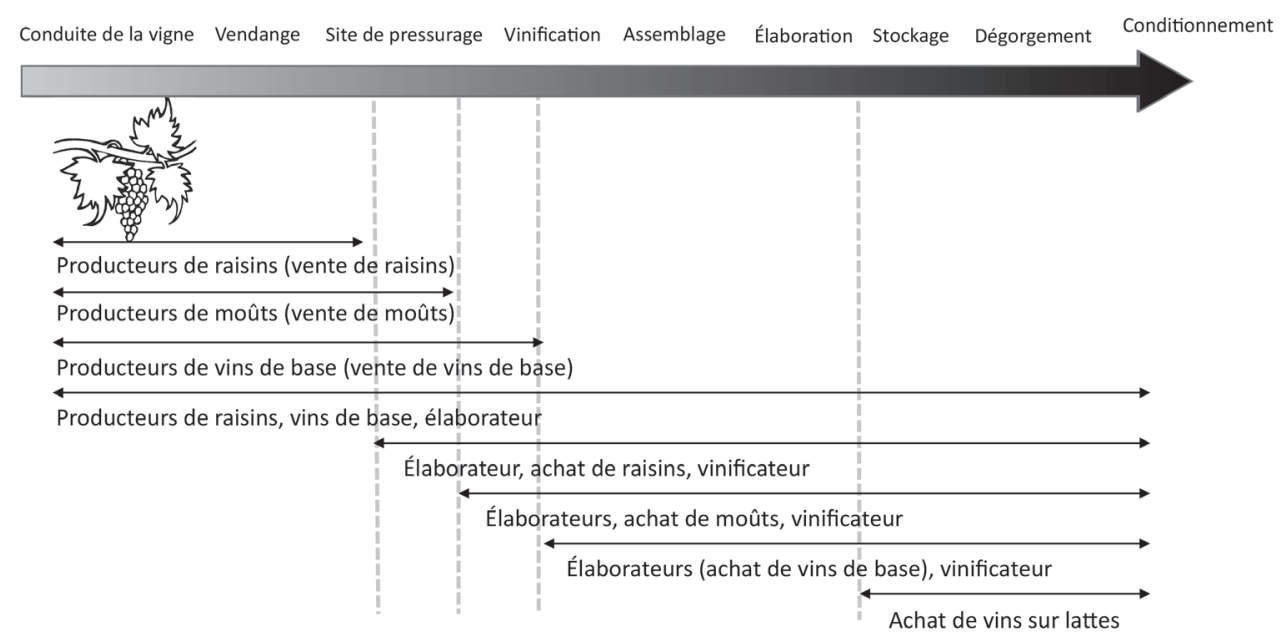

Source : UPECB, 2008.

13 Le moût est du jus de raisin non fermenté obtenu par le pressurage, est destiné à produire du vin par fermentation alcoolique.

14 Voir note 9 : le vin qui n'est pas effervescent est dit « tranquille». 
Si une division du travail s'est instaurée entre coopératives et entreprises privées comme c'est le cas dans le Champagne, les relations entre ces deux groupes sont nombreuses. Les coopératives fournissent le vin de base, ce qui permet de sécuriser une partie de leurs débouchés et de garantir les approvisionnements des élaborateurs. Les élaborateurs fabriquent du Crémant de Bourgogne pour le compte des coopératives qui ainsi complètent leur gamme de vins tranquilles. Les coopératives assurent le lien entre l'interprofession et les viticulteurs qui sont adhérents des coopératives très implantées dans le département de Saône et Loire. Selon Walsh (2012), les coopératives vinifient en effet près de la moitié des vins de Saône de Loire. Elles constituent donc un puissant relai des décisions de régulation et d'amélioration de la qualité de la production que l'interprofession souhaite mettre en place. En effet, de même que les champenoises, les coopératives bourguignonnes encadrent leurs adhérents en leur fournissant des conseils, en particulier techniques. Elles ont ainsi joué récemment un rôle central dans la mise en place d'une innovation organisationnelle : le déploiement d'un nouvel outil de régulation de la production de raisin (l'affectation parcellaire) avant que celui-ci ne devienne obligatoire dans le nouveau cahier des charges de l'AOC.

Une innovation organisationnelle portée par les coopératives: la mise en place d'un outil de régulation collectif de la production dans la filière crémant

La croissance rapide de la production de Crémant de Bourgogne pose de façon aiguë la question de la sécurité des approvisionnements ${ }^{15}$ en raisin des élaborateurs. Dans le vignoble bourguignon, la superposition des zones de production de raisins de différentes appellations fait qu'une parcelle de vigne peut produire du raisin à destination de la production de vin tranquille (appellation régionale) ou effervescent (appellation Crémant). Le viticulteur peut, selon les années en fonction de différents paramètres (rendement, maturité du raisin, conditions climatiques, prix du raisin, etc.), destiner sa production de raisins à l'appellation qui valorisera le mieux son produit. Afin de limiter les comportements opportunistes des viticulteurs qui attendent le plus longtemps possible avant de décider de l'affectation de leurs raisins, l'interprofession du Crémant a souhaité intégrer un outil de régulation de la production de raisin applicable sur le vignoble : l'affectation parcellaire. Il s'agit d'une déclaration qui doit être faite par les viticulteurs avant le 31 mars, sur l'identification des parcelles, dont la production est destinée à l'élaboration de Crémant de Bourgogne. Cette disposition est intégrée dans le cahier des charges (Décret du 19 octobre 2009).

La déclaration d'affectation parcellaire, qui constitue au sein de l'interprofession une innovation organisationnelle, doit mener à une amélioration de la qualité des approvisionnements par la conduite spécifique du vignoble et permettre de mieux anticiper les quantités de raisins destinées à l'élaboration de Crémant de Bourgogne.

La volonté des coopératives de mieux valoriser le raisin de leurs adhérents passera dans les années à venir par l'élaboration complète du Crémant. Aujourd'hui, une minorité de coopératives intègre l'ensemble des phases du processus de production du Crémant. Cette évolution réduira la quantité de vin de base disponible et fragilisera les élaborateurs qui n’ont

15 Le président de l'interprofession indique que son premier objectif est la sécurisation des approvisionnements, c'est-à-dire qu'il convient de faire en sorte de trouver des systèmes pour avoir une production régulière (le journal de Saône et Loire du 10/03/2014). 
pas sécurisé leur approvisionnement. Une minorité d'élaborateurs possède d'ailleurs un domaine leur permettant de produire une partie du raisin (entre 30 à $70 \%$ de leurs besoins selon les entreprises). Pour les autres, c'est l'achat de raisins ou de vins de base en passant des contrats avec les coopératives ou des viticulteurs qui couvre la totalité de leurs besoins.

Depuis 2012, les disponibilités en raisins ou vins de base sont insuffisantes pour faire face aux besoins des élaborateurs. L'interprofession (UPECB) s'attend à perdre des marchés faute des matières premières disponibles. La régularisation de la production est donc stratégique pour la croissance et la montée en gamme de la production de Crémant. La déclaration d'affectation parcellaire est un outil permettant d'atteindre cet objectif collectif à la filière.

\subsubsection{LA COCEBI, UNE PIONNIÈre DANS LE DÉVELOPPEMENT DES CÉRÉALES BIO}

Un outil au service d'un projet de création d'une filière de céréales biologiques

La Cocebi (Coopérative agricole de céréales bio), créée en 1983 à Nitry dans le département de l'Yonne en Bourgogne, est la première coopérative française de céréales biologiques. À cette époque, sept agriculteurs décident de constituer une coopérative afin d'organiser l'offre et la mise en marché dans le but de commercialiser leurs produits bio.

Au début des années quatre-vingt, la création d'une coopérative bio exigeait des innovations organisationnelles (structuration d'une filière, des circuits de distribution et commercialisation...) et constituait elle-même une innovation organisationnelle indispensable pour ces agriculteurs afin de commercialiser leur production. En effet, la profession céréalière et les opérateurs (coopératives de collecte " conventionnelles ", moulins, etc.) étaient hostiles à l'idée même de production céréalière biologique. Si les mentalités ont depuis évolué, il faut constater qu'encore aujourd'hui, la production et la commercialisation de céréales bio restent très marginales. Ainsi, les surfaces en production bio ne représentent que $1,4 \%$ des surfaces totales en céréales alors que les superficies totales en production bio s'élèvent à 845440 ha en 2010, soit 3,1\% de la surface agricole de France (Agence Bio 20116). Par ailleurs, les principales régions céréalières (Bassin parisien, Est et Nord de la France) "présentent un taux réduit de conversions suite à l'absence de structures de développement et d'approvisionnement "relais" et à la concurrence des productions spécialisées à forte valeur ajoutée (pomme de terre, betterave, etc.) auxquelles s'ajoute un certain rejet professionnel de cette agriculture alternative face à un système conventionnel dominant. Ces régions qui réalisent plus de $44 \%$ de la collecte de blé tendre conventionnel n'assurent plus que $5 \%$ de la collecte de blé tendre biologique. » (Dobson, Breslin, Suckley, Barton et Rodriguez, 2013).

\section{L’idéal coopératif au service de l'innovation}

La coopérative Cocebi a innové sur le plan organisationnel afin de promouvoir un projet d'écodéveloppement agricole et rural en rupture avec le modèle d'agriculture productiviste. Pour mettre en place ce projet, elle s'appuie sur une organisation de type "idéal coopératif» qui la distingue d'autres structures coopératives. En effet, le lien entre la coopérative et ses

16 http://www.agencebio.org/la-bio-en-france. 
adhérents est fort (d’après les entretiens menés), et les agriculteurs gèrent une partie du stockage et de la transformation en contrepartie d'une aide financière. Certaines activités de la coopérative comme la gestion, le transport et l'identification des lots sont même assurés par les coopérateurs sous forme de vacations. Il n'y a pas de séparation entre les adhérents et la sphère de décision comme dans les grandes structures coopératives.

La coopérative œuvre pour structurer une filière céréale bio durable afin d'éviter les risques de déstructuration du marché, en contractualisant et planifiant une partie de sa production. C'est le cas, par exemple, du partenariat avec le réseau de magasins Biocoop. « Biocoop contractualise ses volumes pour l'année à venir et donne des estimations et des tendances pour les deux années suivantes. Grâce à cet engagement illustré par la démarche "Ensemble pour plus de sens" à laquelle nous adhérons, nous pouvons anticiper, en termes de quantité, nos besoins de production et nos semences ${ }^{17}$ explique le président de la Cocebi. La contractualisation en volumes s'accompagne d'une fourchette de prix et permet ainsi d'assurer une rémunération équitable pour le producteur.

Par ailleurs, la coopérative apporte un soutien actif à la création d'autres coopératives biologiques dans d'autres régions. Ces actions permettent de développer une organisation indispensable pour le développement des filières céréales biologiques.

La création de la Cocebi a permis de valoriser les céréales biologiques de ses adhérents en structurant les débouchés alors que les opérateurs conventionnels ne considéraient pas à l'époque la production biologique comme viable économiquement. Mais cette structuration de la filière a atteint ses limites du fait, en particulier, de la petite taille des opérateurs et de son faible poids économique.

La mise en place d'une nouvelle filière de valorisation des céréales biologiques : une innovation organisationnelle majeure

En France, le développement de la demande de céréales biologiques est actuellement très important et la production française incapable de la satisfaire. Les transformateurs ou distributeurs importent donc pour faire face à une telle demande des céréales biologiques. D’autre part, si la filière française céréales biologiques se développe, elle reste marginale et à un stade de type " artisanal ». À l'exemple de la Cocebi ${ }^{18}$, les collecteurs de céréales biologiques sont des petites structures. Se pose alors la question de la capacité de ces acteurs à impulser une hausse conséquente de la production et à la valoriser.

La société Decollogne, filiale de la coopérative Dijon Céréales ${ }^{19}$, vient de faire construire un moulin à Aiserey en Côte-d'Or, dont l'activité est de transformer exclusivement des céréales biologiques. Ce moulin est opérationnel depuis 2011. Pour l'approvisionner, la filière régio-

17 http://www.biocoop.fr/actualites-bio/les-filieres-agricoles-bio-les-cereales.

18 La Cocebi compte 171 adhérents, elle emploie dix salariés pour un chiffre d'affaires de 5,2 millions d'euros en 2010. La coopérative a mis en place diverses démarches qualité conduisant à de nombreuses innovations organisationnelles: HACCP, certification ISO 9001, certification CSA.

19 Le groupe coopératif Dijon Céréales emploie plus de 700 salariés sur l'ensemble de ses activités. Son chiffre d'affaires a dépassé les 400 millions d'euros en 2007-2008. La coopérative compte 4000 adhérents. 
nale céréales biologiques doit ainsi passer d'une production actuelle de 6000 tonnes de blé à plus de 20000 tonnes. Le changement d'échelle est important et il faut repenser complètement l'organisation de la filière biologique régionale qui doit être basée sur un projet commun entre différents acteurs régionaux. En effet, il est indispensable que la coopérative soit en mesure de créer un véritable partenariat entre ces acteurs (coopératives, entreprises) afin de valoriser l'ensemble des productions autre que le blé, car la production biologique implique d'adopter une rotation sur longue période des cultures.

Par ailleurs, les coopératives doivent proposer une contractualisation très innovante (qui valorise l'ensemble de ces productions) et suffisamment attractive pour convaincre une partie des céréaliers conventionnels à se convertir à l'agriculture biologique et à s'associer à une démarche commune. Celle-ci nécessite de faire évoluer leur projet individuel qui, très souvent, vise à exploiter au mieux les opportunités d'un marché des céréales très spéculatif.

Synthèse des trois cas :

TABLEAU 2. LES INNOVATIONS ORGANISATIONNELLES MISES EN CEUVRE PAR LES COOPÉRATIVES

\begin{tabular}{|c|c|c|c|c|}
\hline & Objectifs & $\begin{array}{l}\text { Nouveaux } \\
\text { modes de } \\
\text { fonctionnement } \\
\text { dans } \\
\text { l'organisation } \\
\text { des procédures }\end{array}$ & $\begin{array}{l}\text { Nouveaux modes } \\
\text { d'organisation des } \\
\text { relations externes }\end{array}$ & $\begin{array}{l}\text { Évolution de } \\
\text { l'activité des } \\
\text { entreprises }^{20}\end{array}$ \\
\hline Union Auboise & $\begin{array}{l}\text { Fidéliser les } \\
\text { adhérents. } \\
\text { Diversifier les } \\
\text { débouchés. } \\
\text { Meilleure } \\
\text { valorisation. }\end{array}$ & $\begin{array}{l}\text { Démarche } \\
\text { qualité vignoble } \\
\text { pour améliorer } \\
\text { les pratiques } \\
\text { respectueuses de } \\
\text { l'environnement. }\end{array}$ & $\begin{array}{l}\text { Prise de contrôle } \\
\text { de l'entreprise } \\
\text { Devaux et } \\
\text { développement de } \\
\text { la marque Devaux. }\end{array}$ & $\begin{array}{l}\text { Devaux voit en } \\
2013 \text { ses ventes } \\
\text { croître de } 15 \% \\
\text { en volume. }\end{array}$ \\
\hline $\begin{array}{l}\text { Coopératives } \\
\text { de la filière } \\
\text { Crémant }\end{array}$ & $\begin{array}{l}\text { Garantir l'appro- } \\
\text { visionnement } \\
\text { en raisins de la } \\
\text { filière. } \\
\text { Meilleure } \\
\text { valorisation de la } \\
\text { production. }\end{array}$ & $\begin{array}{l}\text { Mise en place } \\
\text { de l'affectation } \\
\text { parcellaire. }\end{array}$ & & $\begin{array}{l}\text { Les coopératives } \\
\text { intègrent de plus } \\
\text { en plus les stades } \\
\text { d'élaboration et } \\
\text { commercialisa- } \\
\text { tion de Crémant. }\end{array}$ \\
\hline
\end{tabular}

20 Les performances d'une entreprise sont le résultat de la combinaison de l'ensemble de ces ressources, il est donc très difficile de déterminer la stricte contribution des innovations aux évolutions de celles-ci. 


\begin{tabular}{|c|c|c|c|c|}
\hline & Objectifs & $\begin{array}{l}\text { Nouveaux } \\
\text { modes de } \\
\text { fonctionnement } \\
\text { dans } \\
\text { l'organisation } \\
\text { des procédures }\end{array}$ & $\begin{array}{l}\text { Nouveaux modes } \\
\text { d'organisation des } \\
\text { relations externes }\end{array}$ & $\begin{array}{l}\text { Évolution de } \\
\text { l'activité des } \\
\text { entreprises }\end{array}$ \\
\hline Cocebi & $\begin{array}{l}\text { Création d'une } \\
\text { filière céréales } \\
\text { biologiques et } \\
\text { accroissement } \\
\text { des débouchés. } \\
\text { Accéder à de } \\
\text { nouveaux mar- } \\
\text { chés auxquels } \\
\text { la coopérative } \\
\text { n'aurait jamais } \\
\text { eu accès aupara- } \\
\text { vant. }\end{array}$ & $\begin{array}{l}\text { HACCP } \\
\text { Certification ISO } \\
9001 . \\
\text { Contractualisa- } \\
\text { tion de la produc- } \\
\text { tion. }\end{array}$ & $\begin{array}{l}\text { Partenariat avec : } \\
\text { — d'autres coopéra- } \\
\text { tives des acteurs } \\
\text { des filières cé- } \\
\text { réalières conven- } \\
\text { tionnelles ; } \\
\text { - d'autres coopé- } \\
\text { ratives bio pour } \\
\text { développer un } \\
\text { pôle semences } \\
\text { bio ; } \\
\text { - Biocoop, pre- } \\
\text { mier réseau de } \\
\text { magasins bio et } \\
\text { équitables en } \\
\text { France ; } \\
\text { - création de } \\
\text { l'union commer- } \\
\text { ciale « Fermes } \\
\text { bio.coop ». }\end{array}$ & $\begin{array}{l}\text { Croissance du } \\
\text { chiffre d'affaires } \\
\text { de près de } 70 \% \\
\text { entre } 2010 \text { et } \\
2015 \text {. }\end{array}$ \\
\hline
\end{tabular}

\section{RÉSULTATS : LA COOPÉRATIVE COMME VECTEUR DE DIFFUSION DES INNOVATIONS}

Partant d'une situation où l'orientation stratégique était axée surtout sur la production, la volonté des coopératives de lutter contre une situation concurrentielle défavorable les a incitées à développer des compétences nouvelles en termes de commercialisation (marques privées et collectives, réseaux, etc.) en rupture avec les routines instituées antérieurement. Les coopératives semblent offrir un contexte facilitant le déploiement des innovations, en particulier organisationnelles. Elles ont établi des relations basées sur des liens de proximité géographique avec leurs adhérents (Bouba-Olga et Grossetti, 2008), mais aussi de proximité organisée. Celle-ci va se traduire par l'adhésion des agriculteurs aux valeurs portées par les coopératives et faciliter ainsi l'acceptation par les coopérateurs de ces innovations (Boschma, 2004 ; Rallet et Torre, 2004). Dans les différents cas étudiés, le développement de compétences a supposé des innovations organisationnelles et de gestion de la production (affectation parcellaire anticipée et nouvelles procédures organisationnelles de formalisation dans le cas du Crémant, développement de circuits de distribution dans les trois cas, partenariats externes... (Tableau 2)) et des réflexions sur le maintien stratégique d'un avantage concurrentiel. Pour les coopératives champenoises comme pour les bourguignonnes, le 
statut coopératif a supposé un développement de compétences, et permis une communication rapide de l'information parmi les adhérents (Mintzberg et Waters, 1984) ainsi qu'une mobilisation facilitée à une stratégie collective (Mintzberg et Waters, 1985 ; Pictet, 2003). Il semble que cette mobilisation a pu reposer sur une confiance des coopérateurs envers l'équipe dirigeante et le bien-fondé de la stratégie qui avait été choisie.

Le cas de la coopérative céréalière, pionnière dans le développement du bio, résulte, il est vrai, d'une histoire bien différente du cas des coopératives spécialisées dans les vins effervescents : la coopérative est constituée de militants, convaincus d'une certaine idéologie écologiste. Ce militantisme s'étend aussi à la forme qu'ils ont choisie pour développer l'entreprise, une forme alors considérée comme alternative, en cohérence avec la raison d'être de leur projet. La différence réside aussi dans l'ampleur du projet, puisqu'il ne s'agit pas ici de modifier l'organisation d'une filière, mais d'en créer une nouvelle de toutes pièces. Mais les points communs sont nombreux : des entrepreneurs "schumpétériens » qui se dressent contre l'ordre économique régnant et les routines établies, innovant par le réseau qu'ils constituent (Biocoop), et construisant, grâce à des innovations organisationnelles, tout l'environnement nécessaire à la réalisation de leur projet. Dans une deuxième phase, la démarche est légitimée par l'environnement socio-politico-économique et sert d'exemple, les partenariats avec de plus grosses structures (Dijon Céréales) se développent.

Finalement, ces trois études de cas nous paraissent avoir mis en évidence plusieurs dimensions :

1. L'évolution des compétences qui a eu lieu et l'importance des innovations organisationnelles nécessaires (gestion de l'affectation parcellaire, mise en place d'une filière complète, développement de nouveaux circuits de distribution et de débouchés), résultat cohérent avec la littérature indiquant un lien entre ces innovations organisationnelles et les compétences autorisant leur mise en œuvre ;

2. L'utilisation de la coopérative comme un réseau de diffusion de connaissances et d'innovations, diffusion qui semble s'être faite dans un contexte de confiance et d'adhésion des adhérents aux décisions prises, résultat nous paraissant intéressant et devoir être approfondi dans des recherches ultérieures ;

3. Les aspects de proximités organisationnelle et cognitive (Boschma, 2005) ${ }^{21}$ qui, à défaut d'une proximité géographique, ont rapproché deux régions produisant le même type de vin autour d'une coopération trans-territoriale, ou des acteurs disséminés dans deux filières céréalières, mais œuvrant pour le développement de l'agriculture biologique.

21 Ron Boschma (2005) a étendu les formes de proximité en se référant à la proximité organisationnelle, la proximité institutionnelle, la proximité géographique, mais aussi la proximité sociale et la proximité cognitive. De manière à faciliter les échanges d'informations et l'absorption de nouvelles connaissances, la distance cognitive entre les acteurs en termes de compétences et savoirfaire ne doit pas être trop grande, mais elle ne doit pas non plus être trop faible, sous peine pour une entreprise d'être en situation de verrouillage (lock-in) sans profiter des opportunités de nouvelles connaissances présentes dans son environnement. 


\section{CONCLUSION : ENSEIGNEMENTS ET LIMITES DE LA RECHERCHE}

Notre question de recherche était centrée sur l'analyse des innovations organisationnelles (en interne d'une part et en termes de partenariats externes d'autre part) et de l'influence du statut des coopératives comme facilitateur de ces innovations du fait des proximités géographiques et organisées avec leurs adhérents. Compte tenu de l'importance donnée par la littérature à la capacité d'insertion dans des collaborations et de partage de l'information pour l'innovation des PME (Nonaka, 1994 ; Julien, Andriambeloson et Ramangalahy, 2004 ; Puthod et Thévenard-Puthod, 2006), il nous semblait intéressant d'explorer le cas des coopératives, qui, par nature, présentent une spécificité, leurs liens privilégiés avec leurs adhérents (Novkovic, 2008). Dans les trois études de cas, la nature coopérative des entreprises impliquées semble bien avoir eu une forte influence sur la façon, dont elles ont su réagir face à une évolution perçue comme négative de leur situation en mettant en œuvre de nouvelles pratiques organisationnelles, voire de nouvelles organisations auxquelles ont adhéré les coopérateurs. Dans les différentes situations, elles ont remis en cause un ordre établi, qui leur était devenu défavorable, et c'est dans leur nature coopérative grâce à l'engagement des adhérents qu'elles disent en particulier avoir trouvé l'énergie et les ressources leur permettant de mener ces changements. Cette nature coopérative leur a permis en effet d'une part de partager l'information et les connaissances nécessaires aux innovations organisationnelles qu'elles voulaient développer. D’autre part, elle les a autorisées à avoir recours plus naturellement que d'autres entreprises de même taille à la constitution ou à l'activation de partenariats notamment avec d'autres coopératives, grâce auxquels elles ont puisé les connaissances et compétences qui leur manquaient.

Ces premiers résultats, encore exploratoires, confirment selon nous l'intérêt de notre question de recherche. Ils nous paraissent encourageants à plusieurs niveaux.

Tout d'abord, nous avons vu que les coopératives agricoles et agroalimentaires, même de petite taille, sont capables d'innovations, parfois ambitieuses, avec des dimensions organisationnelles et marketing importantes. Autrement dit, le lien fort d'une coopérative avec son territoire ne l'empêche pas d'innover.

Plus encore, il nous semble que loin de les empêcher d'innover, les caractéristiques de ces coopératives ont pu, par certains aspects, se révéler des avantages pour innover, grâce au maintien de liens étroits avec les adhérents, à une habitude de collaborer avec des partenaires en particulier d'autres coopératives, à une rapidité dans le partage d'information. On retrouve souvent ces caractéristiques clés dans le cas d'innovations développées par des PME classiques (Puthod et Thévenard-Puthod, 2006). Les coopératives étudiées ont ici fait preuve de compétences particulières dans ces dimensions partenariales et dans la motivation collective de leurs adhérents, qui leur ont permis d'accomplir des innovations organisationnelles rendues nécessaires par leur situation concurrentielle.

Cette recherche exploratoire nous a encouragés à approfondir la compréhension de ces processus d'innovations organisationnelles dans des entreprises coopératives. Les dimensions de confiance entre les coopérateurs et les décideurs, et d’adhésion qui semblent avoir permis une diffusion rapide des informations et des pratiques doivent être explorées de façon plus précise, d'autant que ces liens privilégiés peuvent être remis en cause dans le contexte de fusion et d'agrandissement des coopératives (Mauget, 2005). En effet, si coopérateurs et coo- 
pérative ont des intérêts communs, ces liens privilégiés peuvent être remis en cause du fait notamment de la croissance de la taille des coopératives par fusion ou adhésion à une union. Les coopératives doivent donc réussir à concilier croissance et maintien, voire renforcement, de l'engagement des coopérateurs. La stratégie d'innovation qu'elles mettent en place a ainsi un double objectif, permettre leur développement économique, mais aussi maintenir les liens de confiance avec leurs adhérents.

Nous avons pu étudier les résultats de ces innovations dans nos trois cas, mais le processus d'innovation lui-même et ses relations avec le modèle coopératif restent à analyser de façon plus approfondie. Un élargissement de l'étude à des coopératives d'autres régions européennes ou du monde pourrait aussi nous aider à approfondir cette question. Si nos études de cas restent plus une invitation à poursuivre cette étude que des résultats à généraliser, elles ont néanmoins le mérite de nous conforter dans cette direction et de nous aider à mieux appréhender la complexité des phénomènes à l'œuvre. L'évolution de la situation économique en général et de l'agriculture française en particulier, ne peut que renforcer l'urgence d'une meilleure compréhension en vue d'un meilleur accompagnement du développement d'entreprises ancrées territorialement dans une tradition en renouvellement.

\section{RÉFÉRENCES}

Akrich, M., Callon, M. et Latour, B. (1988). À quoi tient le succès des innovations ? 2 : le choix des porte-parole. Gérer et Comprendre, Annales des Mines, 1988(12), 14-29.

Allaire, G. et Sylvander, B. (1997). Qualité spécifique et innovation territoriale. Cahiers d'Économie et Sociologie Rurales, 1997(44), 29-59.

Ambiaud, E. (2009). Dans la transformation ou le commerce de gros : 2500 coopératives agroalimentaires. Agreste Primeur, (220), 4 p.

Arcas-Lario, N. et Hernandez-Espallardo, M. (2003). Co-ordination and performance of Spanish second-level agricultural co-operatives : the impact of relationship characteristics. European Review of Agricultural Economics, 30(4), 487-507.

Ayerbe-Machat, C. (2006). Innovation technologique et organisationnelle au sein de PME innovantes : complémentarité des processus, analyse comparative des mécanismes de diffusion. Revue internationale PME, 19(1), 9-34.

BARney, J.B. (1991). Firm resources and sustained competitive advantage. Journal of Management, 17(1), 99-120.

BARney, J.B. et Clark, D.N. (2007). Resource-based theory : creating and sustaining competitive advantage. Oxford, Oxford University Press.

Barraud-Didier, V. et Henninger, M.-C. (2009). Les déterminants de la fidélité des adhérents de coopératives agricoles. Revue internationale de l'économie sociale 2009(314), 1-15.

Barraud-Didier, V., Henninger, M.-C. et Anzalone, G. (2013). La distanciation de la relation adhérent-coopérative en France. Études rurales, 2(190), 119-130.

Barraud-Didier, V., Henninger, M.-C. et Triboulet, P. (2014). La participation des adhérents dans leurs coopératives agricoles : une étude exploratoire du secteur céréalier français. Canadian Journal of Agricultural Economics/Revue canadienne d'agroeconomie, 62(1), 125-148. 
Basterretxea, I. et Martínez, R. (2012). Impact of management and innovation capabilities on performance : are cooperatives different? Annals of Public and Cooperative Economics, 83(3), 357-381.

Bélis-Bergouignan, M.-C., Corade, N., Couret, F. et Pesme, J.-O. (2007). Le vignoble aquitain, entre atomicité et concentration. Cahiers de Recherche du CEREBEM, 2007(112-07), 3-29.

Ben Letaifa, S. et Rabeau, Y. (2013). Too close to collaborate ? How geographic proximity could impede entrepreneurship and innovation. Journal of Business Research, 66(10), 2071-2078.

Birchall, J. (2011). People-centred businesses: co-operatives, mutuals and the idea of membership. Londres/New York, Palgrave MacMillan.

BIRLEY, S. (1985). The role of network in the entrepreneurial process. Journal of Business Venturing, 1(1), 107-117.

Boeme, D.M. (2013). Collaborate at home to win abroad : how does access to local network resources influence export behavior? Journal of Small Business Management, 51(2), 167-182.

Boschma, R. (2004). Proximité et innovation. Économie rurale, 1(280), 8-24.

Boschma, R. (2005). Proximity and innovation : a critical assessment. Regional Studies, 39(1), 61-74.

Bouba-Olga, O. et Grossetti, M. (2008). Socioéconomie de proximité. Revue d'Économie Régionale et Urbaine, (3), 311-328.

Bourgeois, L.J. et Brodwin, D.R. (1984). Strategic implementation : five approaches to an elusive phenomenon. Strategic Management Journal, 5(3), 241-264.

Charreire, S. et Durieux, F. (1999). Explorer et tester : les deux voies pour la recherche. Dans R. Thiétart (dir.), Méthodes de recherche en management (p. 57-81). Paris, Dunod.

Chatenier, E.D., Verstegen, J.A.A.M., Biemans, H.J.A., Mulder, M. et Omta, O.S.W.F. (2010). Identification of competencies for professionals in open innovation teams. R\&D Management, 40(3), 271-280.

Chetty, S. (1996). The case study method for research in small and medium-sized firms. International Small Business Journal, 15(1), 73-85.

Chiffoleau, Y., Dreyfus, F., Ewert, J., Martin, C., Touzard, J.-M. et Williams, W. (2002). Qualité et solidarité dans les coopératives viticoles : des enjeux communs en Afrique du Sud et en Languedoc. Revue internationale de l'économie sociale, (285), 63-74.

CIVC (2010). Observation économique. Tableau de bord de la filière Champenoise, juillet 2010. Comité interprofessionnel des vins de Champagne. Récupéré le 5 mai 2016 du site : http://extranet.comitechampagne.fr/EcoStats/Documents/TAB22009Extranet\%20FINAL.pdf.

Cohen, W.M. et Levinthal, D.A. (1989). Innovation and learning : the two faces of R\&D. The Economic Journal, 99(397), 569-596.

Cohen, W.M. et Levinthal, D.A. (1990). Absorptive capacity : a new perspective on learning and innovation. Administrative Science Quarterly, 35(1), 128-152.

D’Angelo, A., Majocchi, A., Zucchella, A. et Buck, T. (2013). Geographical pathways for SME internationalization : insights from an Italian sample. International Marketing Review, 30(2), 80-105.

Divry, C., Dubuisson, S. et Torre, A. (1999). Une caractérisation des compétences par les formes d'apprentissage. Dans D. Foray et J. Mairesse, Innovation et performances - Approches interdisciplinaires (p. 261-281). Paris, Éditions de l’École des hautes études en sciences sociales. 
Dobson, S., Breslin, D., Suckley, L., Barton, R. et Rodriguez, L. (2013). Small firm survival and innovation : an evolutionary approach. International Journal of Entrepreneurship \& Innovation, 14(2), 69-80.

Dosi, G. (1988). Sources, procedures, and microeconomic effects of Innovation. Journal of Economic Literature, 26(3), 1120-1171.

Dosi, G., Teece, D. et Winter, S. (1990). Les frontières des entreprises : vers une théorie de la cohérence de la grande entreprise. Revue d'économie industrielle, (51), 238-254.

Dussuc, B. et Geindre, S. (2012). Capital social, théorie des réseaux sociaux et recherche en PME : une revue de la littérature. Actes du colloque CIFEPME 2012. Brest, France, octobre.

EIsenhardt, K. (1989). Building theory from case study research. Academy of Management Review, 14(4), 532-550.

Esteve-Pérez, S. et Rodríguez, D. (2013). The dynamics of exports and R\&D in SMEs. Small Business Economics, 41(1), 219-240.

Fensterseifer, J.E. et Rastoin, J.-L. (2013). Cluster resources and competitive advantage. International Journal of Wine Business Research, 25(4), 267-284.

FILIPPI, M. (2004). Réorganisations dans la coopération agricole : proximités et solidarité territoriale. Économie rurale, 280(1), 42-58.

Filippi, M. et Triboulet, P. (2006). Typologie des comportements à innover des coopératives agricoles, une étude en région Midi-Pyrénées. Économie rurale, 6(296), 20-35.

Fonrouge, C. (2008). Entrepreneuriat et innovations organisationnelles, pratiques et principes. Revue française de gestion, 5(185), 107-123.

Freel, M.S. (2003). Sectoral patterns of small firm innovation, networking and proximity. Research Policy, 32(2003), 751-770.

Fulton, M. (1999). Cooperatives and member commitment. The Finnish Journal of Business Economics, 4(99), 418-437.

GAdE, D.W. (2004). Tradition, territory, and terroir in French viniculture : Cassis, France, and appellation contrôlée. Annals of the Association of American Geographers, 94(4), 848-867.

Galliano, D., Garedew, L. et Magrini, M.-B. (2011). Les déterminants organisationnels de l'innovation produit : les spécificités des firmes agroalimentaires françaises. Dans N. Merle-Lamoot et G. Pannetier (dir.), L'innovation dans les entreprises. Moteurs, moyens et enjeux (p. 50-68). Paris, DGCIS Analyses.

Giuliani, E. et Bell, M. (2005). The micro-determinants of meso-level learning and innovation : evidence from a Chilean wine cluster. Research Policy, 34(1), 47-68.

Julien, P.-A., Andriambeloson, E. et Ramangalahy, C. (2004). Networks, weak signals and technological innovations among SMEs in the land-based transportation equipment sector. Entrepreneurship and Regional Development, 16(4), 251-269.

Lam, A. (2004). Organizational innovation. Munich, University of Muenchen.

Levi, Y. et Pellegrin-Rescia, M.L. (1997). A new look at the embeddedness/disembeddedness issue : cooperatives as terms of reference. Journal of Socio-Economics, 26(2), 159-179. 
Mangin, P. (2012). L’entreprise coopérative : un modèle anti-crise. Le Monde. Récupéré le 14 mai 2014 sur le site : http://www.lemonde.fr/idees/article/2012/03/05/l-entreprise-cooperative-un-modele-anticrise_1650928_3232.html.

Marchesnay, M. (2001). Les PME de terroir : entre « geo » et «clio » stratégies. Entreprises et histoire, 28(2), 51-63.

Martin, M. et TAnguy, C. (2011). Le rôle de l'innovation organisationnelle dans les petites entreprises : éclairages à partir du secteur agroalimentaire. Dans A. Hamdouch, S. Reboud et C. Tanguy (dir.), PME, dynamiques entrepreneuriales et innovation (p. 251-276). Bruxelles, Peter Lang.

Martin, R. et Boschma, R. (2010). The Handbook of evolutionary economic geography. Cheltenham, Edward Elgar.

Mauget, R. (2005). Les coopératives agroalimentaires face aux enjeux de la mondialisation. Revue internationale de l'économie sociale, (297), 42-59.

Mauget, R. (2013). Stratégies et restructurations des coopératives dans la mondialisation des marchés. Dans C. Chomel, F. Declerck, M. Filippi, O. Frey et R. Mauget (dir.), Les coopératives agricoles - Identité, gouvernance et stratégies. Bruxelles, Éditions Larcier.

Mintzberg, H. et Waters, J.A. (1984). Of strategies, deliberate and emergent. Readings in Strategic Management. Milton Keynes, Open University Press.

Mintzberg, H. et Waters, J.A. (1985). Of strategies, deliberate and emergent. Strategic Management Journal, 6(3), 257-272.

Nelson, R. et Winter, S. (1982). An evolutionary theory of economic change. Cambridge, Massachusetts, Harvard University Press.

NonAKA, I. (1994). A dynamic theory of organizational knowledge creation. Organizational Science, 5(1), 14-37.

Novkovic, S. (2008). Defining the co-operative difference. Journal of Socio-Economics, 37(6), 21682177.

OCDE (2005). Oslo manual : guidelines for collecting and interpreting technological innovation data. Oslo, Organisation of Economic Co-operation and Development.

Picteт (2003). The watch industry: what makes it tick? Genève, Pictet and Cie.

Puthod, D. et Thévenard-Puthod, C. (2006). Coopération, tensions et conflit dans un réseau d'innovation construit autour d'une PME. Revue française de gestion, 5(164), 181-204.

Rallet, A. et Torre, A. (2004). Proximité et localisation. Économie rurale, 280(1), 25-41.

RAstoin, J.-L. et VissaC-Charles, V. (1999). Le groupe stratégique des entreprises de terroir. Revue internationale PME, 12(1-2), 193-200.

Rogers, E.M. (1995). Diffusion of innovations. New York, The Free Press.

Salavou, H., Baltas, G. et Lioukas, S. (2004). Organisational innovation in SMEs : the importance of strategic orientation and competitive structure. European Journal of Marketing, 38(9-10), 1091-1112.

Shiraishi, M. (2009). The function of the food policy and the co-operative as NPO sector under the new direction of the food system. Journal of Agriculture Science, 53(4), 305-310.

Sohal, A., Simon, A. et Lu, E. (1996). Generative and case study research in quality management Part II : practical examples. International Journal of Quality \& Reliability Management, 13(2), 75-87. 
Stervinou, S. et LÊ, S. (2006). Une méthodologie pour analyser les groupements localisés d'entreprises : le cas de l'association Produit en Bretagne. $8^{e}$ CIFEPME, L'internationalisation des PME et ses conséquences sur les stratégies entrepreneuriales. Fribourg, Suisse, 25-27 octobre 2006.

Street, C.T. et Cameron, A.F. (2007). External relationships and the small business : a review of small business alliance and network research. Journal of Small Business Management, 45(2), 239-266.

Teece, D.J. (1986). Profiting from technological innovation : implications for integration, collaboration, licensing and public policy. Research Policy, 15(6), 285-305.

Teece, D.J. (1988). Technological change and the nature of the firm. Dans C. Freeman, R. Nelson, G. Silverberg et L. Soete (dir.), Technical change and economic theory (p. 256-281). Londres/New York, Pinter Publishers.

Teece, D.J. (1998). Research directions for knowledge management. California Management Review, 40(3), 289-293.

Ter Wal, A. et Boschma, R. (2011). Co-evolution of firms, industries and networks in space. Regional Studies, 45(7), 919-933.

TORre, A. (2010). Jalons pour une analyse dynamique des proximités. Revue d'Économie Régionale et Urbaine, (3), 409-437.

Torre, A. et Rallet, A. (2005). Proximity and localization. Regional Studies, 39(1), 47-59.

UPECB, C., CAVI, ICONE (2008). Plan de contrôle pour l'appellation Crémant de Bourgogne. 60 diapositives. Présentation du 17 novembre 2008.

WALSH, P.R. (2012). Innovation Nirvana or innovation Wasteland ? Identifying commercialization strategies for small and medium renewable energy enterprises. Technovation, 32(1), 32-42.

Williamson, O.E. (1979). Transaction-cost economics : the governance of contractual relations. Journal of Law and Economics, 22(2), 233-261.

YIN, R.K. (1989). Case study research : design and methods. Londres, Sage Publications.

Zimmermann, J.-B. (2008). Le territoire dans l'analyse économique. Revue française de gestion, 4(184), 105-118. 